Research Paper

\title{
The antipsychotic agent flupentixol is a new PI3K inhibitor and potential anticancer drug for lung cancer
}

\author{
Chao Dong1", Yin Chen2\# , Hongjian Li33,4, Yi Yang5, Hongtao Zhang6\#, Kunbin Ke², Xi-Nan Shi , Xu Liu ${ }^{8}$, \\ Ling Li ${ }^{8}$, Jing Ma9 ${ }^{9}$ Hsiang-Fu Kung ${ }^{8}$, Ceshi Chen ${ }^{10}$, Marie Chia-mi Lin ${ }^{11^{凶}}$ \\ 1. Department of the second medical oncology, The 3rd Affiliated Hospital of Kunming Medical University, Yunnan Tumor Hospital, Kunming, China \\ 2. Department of Urology, the 1st Affiliated Hospital of Kunming Medical University, Kunming, China \\ 3. SDIVF R\&D Centre, Hong Kong Science Park, Sha Tin, New Territories, Hong Kong. \\ 4. CUHK-SDU Joint Laboratory on Reproductive Genetics, School of Biomedical Sciences, The Chinese University of Hong Kong, Sha Tin, New Territories, \\ Hong Kong. \\ 5. Department of the radiation oncology, The 3rd Affiliated Hospital of Kunming Medical University, Yunnan Tumor Hospital, Kunming, China. \\ 6. Department of the colorectal surgery, The 3rd Affiliated Hospital of Kunming Medical University, Yunnan Tumor Hospital, Kunming, China. \\ 7. Department of Pathophysiology, School of Basic Medical Sciences, Yunnan University of TCM, Kunming, China. \\ 8. Biomedical Engineering Research Center, Kunming Medical University, Kunming, Yunnan, China \\ 9. Department of Otolaryngology, Head and Neck Surgery, Kunming Children's Hospital, Kunming, China. \\ 10. Key Laboratory of Animal Models and Human Disease Mechanisms of Chinese Academy of Sciences and Yunnan Province, Kunming Institute of Zoology, \\ Chinese Academy of Sciences, Kunming, Yunnan, China \\ 11. Institute of Medical and Pharmaceutical Sciences, The Academy of Medical Science, Zhengzhou University, Zhengzhou, China.
}

\# These authors contributed equally to this work.

$\square$ Corresponding authors: Marie Chia-mi, Institute of Medical and Pharmaceutical Sciences, The Academy of Medical Science, Zhengzhou Uninversity, Science Avenue 100, Zhnegzhou, Henan, 450001, China. Tel: +86-37-9834-6647; e-mail: mcmlin@163.com and Ceshi Chen, Key Laboratory of Animal Models and Human Disease Mechanisms of Chinese Academy of Sciences, Kunming Institute of Zoology, Chinese Academy of Sciences, 32 Jiaochang East Road, Kunming, Yunnan, 650223, China. Tel: +86-871-65181944; Fax: +86--871-65181945; e-mail: chenc@mail.kiz.ac.cn

(c) Ivyspring International Publisher. This is an open access article distributed under the terms of the Creative Commons Attribution (CC BY-NC) license (https://creativecommons.org/licenses/by-nc/4.0/). See http://ivyspring.com/terms for full terms and conditions.

Received: 2018.12.28; Accepted: 2019.04.10; Published: 2019.06.02

\begin{abstract}
Background: The phosphatidylinositol 3-kinase (PI3K)/AKT signaling pathway is hyperactivated in lung cancer and regulates a broad range of cellular processes, including proliferation, survival, angiogenesis, and metastasis. Thus PI3K is considered a promising target for therapy. To date, PI3K inhibitors have not been approved for lung cancer. Recent studies showed that the antipsychotic agent flupentixol induced apoptosis of lung cancer cell, however the anti-tumor mechanism of flupentixol remains unclear.

Methods: (1) The idock software simulated the molecular docking between the PI3Ka protein and flupentixol. (2) Inhibition of PI3K $\alpha$ by the flupentixol was examined by in vitro kinase assays. (3) The cytotoxicity of flupentixol on the NSCLC cell lines was tested by MTT assays. (4) We treated A549 and $\mathrm{H} 661$ cells with flupentixol and then measured the percentage of apoptotic cells by the Annexin $\mathrm{V} / \mathrm{PI}$ analysis. (5) We investigated the effect of flupentixol on the expression of critical PI3K/AKT signaling pathway proteins, further analyzed on the cleavage of PARP and caspase-3 by Western blotting. (6) BALB/C nude mice were subcutaneously injected with A549 cells to evaluate the effect of flupentixol on the growth of lung carcinoma.

Results: Structural analysis of the predicted binding conformation suggested that flupentixol docks to the ATP binding pocket of PI3Ka. Kinase assays demonstrate that flupentixol indeed inhibited the PI3K $\alpha$ kinase activity. Flupentixol exhibited cytotoxicity in lung cancer cell lines A549 and H661 in a dose- and time-dependent manner. Furthermore, flupentixol more strongly inhibited the phosphorylation of AKT (T308 and S473) and the expression of its downstream target gene Bcl-2 than two known PI3K inhibitors (BYL719 and BKM120). Flupentixol induced apoptosis as measured by PARP and caspase- 3 cleavage. Finally, flupentixol significantly suppressed A549 xenograft growth in BALB/C nude mice.
\end{abstract}


Conclusions: Flupentixol could be docked to the PI3K $\alpha$ protein and specifically inhibit the $\mathrm{PI3K} / \mathrm{AKT}$ pathway and survival of lung cancer cells in vitro and in vivo. As an old drug, flupentixol is a new PI3K inhibitor that may be used for the treatment of lung cancers.

Key words: Flupentixol, PI3K inhibitor, PI3Ka, Lung cancer

\section{Introduction}

Nonsmall-cell lung cancer (NSCLC) is the leading cause of cancer deaths worldwide [1]. In the last decade, the identification of activating EGFR mutations and ALK rearrangements allowed new targeted therapies and greatly improved the survival and prognosis of patients with advanced NSCLC [2]. However, drug resistance is a great challenge for targeted therapies, such as EGFR and ALK inhibitors $[3,4]$. Therefore, it is urgent to explore drug resistance mechanism and identify new therapeutic targets for the combination therapy.

The phosphatidylinositol 3-kinase (PI3K)/AKT signaling pathway is hyperactivated in lung cancer and regulates a broad range of cellular processes, including proliferation, survival, angiogenesis, and metastasis [5]. Activation of this signaling pathway has been frequently associated with drug resistance [6]. Lung cancer patients' acquired resistance to EGFR inhibitors usually is attributed to the activation of the PI3K/AKT pathway [7]. PI3K pathway inhibitors have emerged as a possible solution for EGFR inhibitor resistance [8]. The PI3K/AKT pathway is frequently activated due to the mutation of PIK3CA, a gene encoding the p110a catalytic subunit of PI3K (PI3K p110a) or the inactivation of the phosphatase and tensin homolog (PTEN) protein [9]. PI3K, as a proto-oncogene, is a promising therapeutic target for lung cancer [10].

Flupentixol is a highly effective antipsychotic drug for the treatment of schizophrenia or depression. Flupentixol was used to treat depressive cancer patients and achieved good results [11]. Flupentixol has been reported to reverse drug resistance and to exhibit a synergetic effect with gefitinib. Recent studies showed that flupentixol induced apoptosis of T790M mutation lung cancer cell and synergized with gefitinib in response to EGFR inhibitor resistance [12]. The chemosensitivity effect of flupentixol resulted from increased intracellular drug accumulation via a mechanism unrelated to P-glycoprotein [13]. However, the anti-tumor mechanism of flupentixol remains unclear.

In this study, we found that flupentixol could be docked to PI3Ka by computer protein-ligand docking software, idock. We further confirmed that flupentixol inhibited cell proliferation and induced apoptosis in two NSCLC cell lines by blocking the PI3K/AKT signaling pathway. Flupentixol may serve as a new PI3K inhibitor for the treatment of lung cancer.

\section{Materials and Methods}

\section{PI3Ka molecular docking by compute ensemble}

The idock software simulated the molecular docking between the PI3Ka protein and flupentixol. The visual docking results are available at http://istar.cse.cuhk.edu.hk/idock/iview/?4JPS-dba p+fda+npc.

\section{Chemicals, antibodies, cell lines, and cell culture}

Flupentixol and two known PI3K inhibitors (BYL719 and BKM120) were purchased from J\&K Scientific Ltd. (Beijing, China). The lung cancer cell lines NCI-H661 (large cell), A549 (adenocarcinoma), NCI-H520 (squamous), and SK-SEM-1 (squamous) were obtained from the Cell Bank of Kunming Institute of Zoology, Chinese Academy of Sciences. The normal lung bronchial epithelial cell line BEAS-2B was obtained from Key Laboratory of Lung Cancer Research in Yunnan Province. NCI-H661 and NCI-H520 cells were cultured in RPMI 1640 containing 10\% fetal bovine serum (FBS) (Invitrogen, Rockville, Maryland, USA). A549 and SK-SEM-1 cells were cultured in DMEM containing 10\% FBS (Invitrogen). BEAS-2B cells were cultured in F-12 containing 10\% FBS (Invitrogen). All media were supplemented with $100 \mathrm{U} / \mathrm{ml}$ penicillin and 0.1 $\mathrm{mg} / \mathrm{ml}$ streptomycin. All cells were cultured at $37^{\circ} \mathrm{C}$ in $5 \% \mathrm{CO}_{2}$.

Antibodies against PI3K-p110a, total AKT, phospho-AKT(S473), phospho-AKT(T308), ERK, phospho-ERK, Bcl-2, VEGF, MMP-9, PARP, pro-caspase3, cleaved-caspase3, and $\beta$-actin were purchased from Cell Signaling Technology (Massachusetts, USA) and Abcam (San Francisco, USA).

\section{Cell-free biochemical kinase inhibition assay}

Inhibition of PI3K $\alpha$ by the flupentixol was examined in a cell-free system by assessing the phosphorylation of a poly-EY (4:1 Glu, Tyr) peptide substrate with recombinant kinases PI3Ka (Upstate Biotechnology). Inhibition of the recombinant kinases 
was evaluated by using the ADP-Glo Kinase assay kit according to the manufacturer's instruction (Promega, Madison, WI, USA). Briefly, the flupentixol in a range of different concentration ( $1 \quad \mathrm{nM}-1 \quad \mu \mathrm{M})$ were incubated with $4 \mathrm{ng}$ of the recombinant kinases and $0.2 \mu \mathrm{g} / \mathrm{mL}$ of the poly-EY substrate at room temperature for $60 \mathrm{~min}$. Then, $5 \mu \mathrm{L}$ of ADP-Glo reagent was added and incubation continued at room temperature for another $40 \mathrm{~min}$. Finally, $10 \mu \mathrm{L}$ of kinase detection reagent was added and the mixture was allowed to incubate at room temperature for 30 min before the measurement of luminescence by GloMax 20/20 Luminometer (Promega).

\section{MTT assays}

A549 and H661 cells were seeded in 96-well plates at a density of $7 \times 10^{3}$ cells per well for 72 hours and treated with $2.5,5,10,20$ and $40 \mu \mathrm{M}$ of chemicals for 24,48 , and 72 hours. The growth inhibitory effects of the chemicals tested on cells were evaluated by MTT assays. Absorbance was measured at $570 \mathrm{~nm}$ with a Synergy 2 microplate reader (Bio-Tek Instruments, Inc., Winooski, VT, USA) according to the standard protocol. The $\mathrm{IC}_{50}$ values were calculated by GraphPad Prism 5 .

\section{Apoptosis analysis by flow cytometry}

A549 and H661 cells were seeded in 24-well plates at a density of $6 \times 10^{4}$ cells per well for 24 hours and exposed to flupentixol, cisplatin, or the combination of the flupentixol and cisplatin for 24 hours. The cells were harvested and stained with Annexin V-FITC/propidium iodide (PI) according to the manufacturer's instructions (Beijing 4A Biotech Co., Ltd, Beijing, China). Apoptotic cells were analyzed by flow cytometry (CyFlow Space/Partec, Germany).

\section{Western blotting}

A549 and H661 cells were seeded in 6-well plates for 24 hours and treated with 10\% FBS containing flupentixol and cisplatin. After 24 hours of incubation, the cells were harvested and lysed with RIPA buffer containing $1 \mathrm{mM}$ PMSF and protease inhibitor cocktail at $4{ }^{\circ} \mathrm{C}$ for 30 minutes and centrifuged at 13,000 rpm for 15 minutes. The supernatants were recovered, and the protein concentrations were measured using the BCA Protein Assay Kit (Thermo Scientific, Massachusetts, USA). The same amounts of cell lysates were resolved by 10\% SDS-PAGE and transferred onto the nitrocellulose membranes (Sigma, Shanghai, China). After blocking with skim milk, the membranes were incubated sequentially with appropriately diluted primary and secondary antibodies. Proteins were detected using the enhanced chemiluminescence detection system (Amersham Biosciences, Piscataway, New Jersey, USA). To calibrate for equal loading of samples, the membranes were reprobed with an anti- $\beta$-actin antibody.

\section{A549 growth in nude mice}

Male BALB/C nude mice of 5-6 weeks old were purchased from the Department of Animal Experiment, Kunming Medical University, and raised under pathogen-free conditions. A549 cells $\left(1 \times 10^{6} / 0.2\right.$ $\mathrm{ml}$ PBS per mice) were injected subcutaneously into the right flank of the mice. Seven days after inoculation, tumors grew to a volume of $80-100 \mathrm{~mm}^{3}$. The mice were randomly divided into two groups (six mice per group) and injected by intragastric injection administration (i.g.) every day for 21 days with either PBS (control group) or flupentixol (40 mg/ $\mathrm{kg}$ in PBS). Tumor volumes were measured every 3-4 days after tumor appearance and calculated by the equation $\mathrm{V}=\mathrm{ab}^{2} / 2$ (a=longest axis; $\mathrm{b}=$ shortest axis). The mice were sacrificed on day 21 after treatment, and tumors were isolated and weighed. The study was approved by the laboratory animal ethics committee of Kunming Medical University.

\section{Statistical analysis}

The results were obtained from at least three different experiments and expressed as the mean \pm SEM (the standard error of the mean). Statistical analysis was performed by one-way ANOVA (analysis of variance), and the difference was considered statistically significant if $\mathrm{p}<0.05$. Statistically significant results are marked with asterisk symbols $\left(^{*}\right)$ in the figures.

\section{Results}

\section{Flupentixol binds to PI3Ka by computer ensemble docking}

In our previous study, we used free and open-source protein-ligand docking software [14, 15] and found that flupentixol binds to the ATP-binding pocket of PI3Ka. Flupentixol dihydrochloride (ZINC ID: 29489118) was found to bind to PI3Ka with a high affinity. Its binding free energy predicted by idock was $-8.52 \mathrm{kcal} / \mathrm{mol}$, and its binding affinity predicted by RF-Score was 7.11 pKD. The structure of flupentixol is shown in Figure 1A. The predicted conformation of flupentixol in complex with PI3Ka is shown in Figure 1B. Putatively, it forms three hydrogen bonds with Val851 and Asp810, a salt bridge with Asp933, and hydrophobic contacts with Tyr836 and Ile932. 
(A)

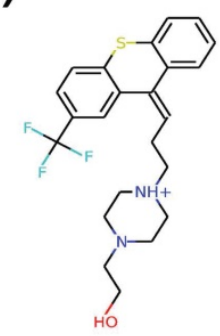

(B)

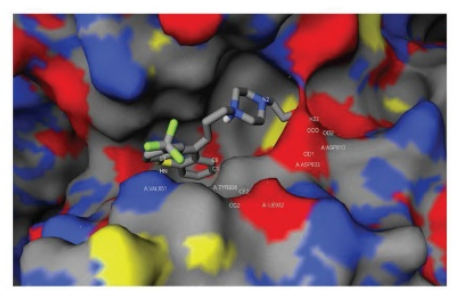

(C)

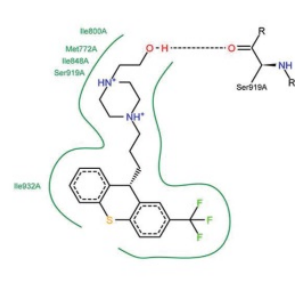

PI3K p110 $\alpha$

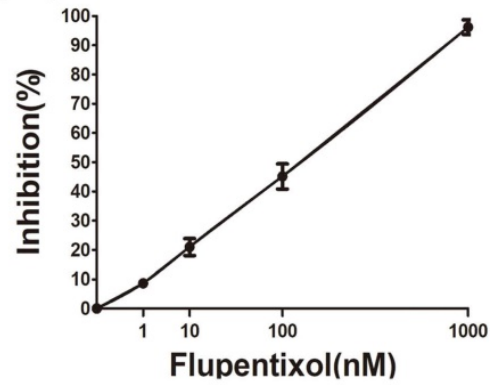

Figure 1. Ensemble docking of flupentixol binding to PI3Ka. (A) The structure of flupentixol. (B) The predicted conformation of flupentixol in complex with PI3Ka. $\mathrm{PI} 3 \mathrm{Ka}$ is shown in molecular surface representation colored by atom type. Flupentixol is rendered as sticks colored by atom type. Intermolecular interacting atoms and residues are labeled. The cyan, purple and green dashed lines indicate hydrogen bonds, salt bridges and hydrophobic contacts, respectively. This figure was created by the web-based visualizer iview. (C) Flupentixol significantly inhibited PI3Ka kinase activity in a dose dependent manner (IC50: $127 \pm 5.87 \mathrm{nM}$ )

\section{Flupentixol significantly inhibited PI3Ka kinase activity in vitro}

Since flupentixol was identified by idock to interact with PI3Ka, we hypothesized that their anti-cancer activity was attributed in part to its ability to inhibit PI3Ka kinase activity. The inhibitory effects of flupentixol on the activity of PI3Ka kinase was evaluated in a cell-free system as described in the method section. The experimental results showed that flupentixol inhibited PI3Ka kinase activity in a dose dependent manner $\left(\mathrm{IC}_{50}=127 \pm 5.87 \mathrm{nM}\right)$ (Figure 1C).

\section{Flupentixol decreases the viability of lung cancer cells}

We tested the cytotoxicity of flupentixol on the NSCLC cell lines including A549, H661, SK-SEM-1, and NCAL-H520 as well as BEAS-2B, an SV40 immortalized normal lung bronchial epithelial cell line by MTT assays. Flupentixol displayed excellent inhibitory effects in four NSCLC cell lines but little cytotoxicity in BEAS-2B cells (Figure 2A). Among the four NSCLC cells, flupentixol exhibited a stronger anticancer effect on A549 and H661 cell lines in a dose- and time-dependent manner $\left(\mathrm{IC}_{50}=5.708 \mu \mathrm{M}\right.$ in A549, 6.374 $\mu \mathrm{M}$ in H661) (Figure 2B). Importantly, the $\mathrm{IC}_{50}$ of flupentixol in A549 and H661 was significantly lower than two known PI3K inhibitors, PI3Ka-selective inhibitor alpelisib BYL719 $\left(\mathrm{IC}_{50}=45.9\right.$ $\mu \mathrm{M})$ and pan-PI3K inhibitor buparlisib BKM120 $\left(\mathrm{IC}_{50}=63.9 \mu \mathrm{M}\right)($ Figure 2C). These results suggest that flupentixol is highly efficient in inhibiting the viability of lung cancer cells.

\section{Flupentixol induces apoptosis in lung cancer cells}

To determine whether flupentixol kills lung cancer cells through apoptosis, we detected apoptosis by the Annexin V/PI analysis. We treated A549 and H661 cells with flupentixol $(5,10,20$ and $40 \mu \mathrm{M})$ for 24 hours and then measured the percentage of apoptotic cells. As shown in Figure 3A, flupentixol (10, 20, or 40 $\mu \mathrm{M})$ significantly increased the percentage of cells in early apoptosis compared with the negative control in both A549 and H661 ( $p<0.05)$ (Figure 3B). We further examined the cleavage of poly-(ADP-ribose)polymerase (PARP) and caspase-3, two apoptotic biomarkers by Western blotting. Flupentixol induced the cleavage of PARP and caspase-3 in a dose-dependent manner (Figure 3C). These results demonstrate that flupentixol induces apoptosis in lung cancer cells.

\section{Flupentixol inhibits p-AKT and $\mathrm{Bcl}-2$ expression levels}

Accumulating evidence indicated that the activation of the PI3K/AKT pathway is involved in lung cancer cell proliferation, survival, invasion, and metastasis. The expression level of phosphorylated AKT (p-AKT) can be used as an indicator to evaluate the inhibition of the PI3K/AKT pathway. We investigated the effect of flupentixol on the expression level of pAKT by Western blotting (Figure 4A). Flupentixol had no effect on PI3K p110a protein expression in $\mathrm{H} 661$ and A549 cells. As expected, AKT phosphorylation (Thr308 and Ser473) levels were significantly decreased by flupentixol in a dose-dependent manner, whereas total AKT protein levels were not affected. Flupentixol did not decrease the phosphorylation levels of ERK, indicating the specificity of flupentixol to the PI3K/AKT pathway. Flupentixol also decreased the expression levels of Bcl-2 but not MMP-9 or VEGF.

Compared to the known PI3K inhibitors (BYL719 and BLM120), the inhibitory effect of flupentixol was stronger in decreasing the expression of AKT phosphorylation and Bcl-2 (Figure 4B).

\section{Flupentixol suppresses A549 xenografted tumor growth in nude mice}

Finally, we evaluated the effect of flupentixol on the growth of lung carcinoma in vivo. BALB/C nude 
mice were subcutaneously injected with A549 cells. Fourteen days after inoculation, tumors grew to a volume of $50-80 \mathrm{~mm}^{3}$. The mice were randomly divided into two groups (six mice per group) and injected by intragastric injection administration (i.g.) every day for 21 days with PBS (control group) or flupentixol $(40 \mathrm{mg} / \mathrm{kg})$. Our results showed that flupentixol significantly reduced tumor volumes compared to the vehicle control $(p<0.05)$ (Figure 5A). Flupentixol also significantly reduced tumor weights by $64.1 \%(p<0.05)$ (Figures 5B-C). The treatment with flupentixol did not substantially affect the average body weight of the mice (Figure 5D). These results suggest that flupentixol is a potentially safe and effective oral anticancer drug for lung cancer.

\section{(A)}

(B)

A549

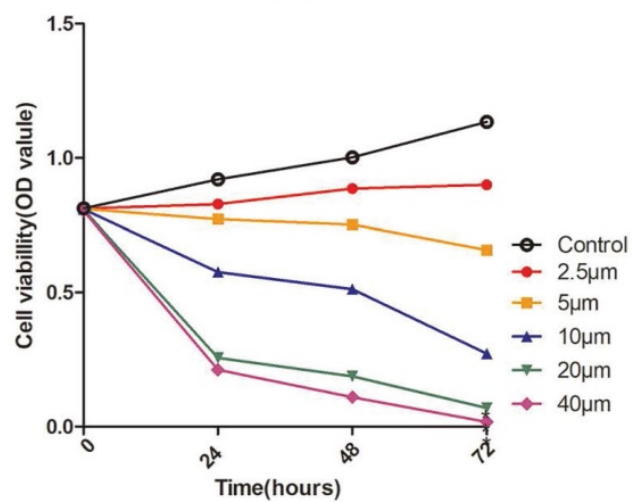

(C)

A549

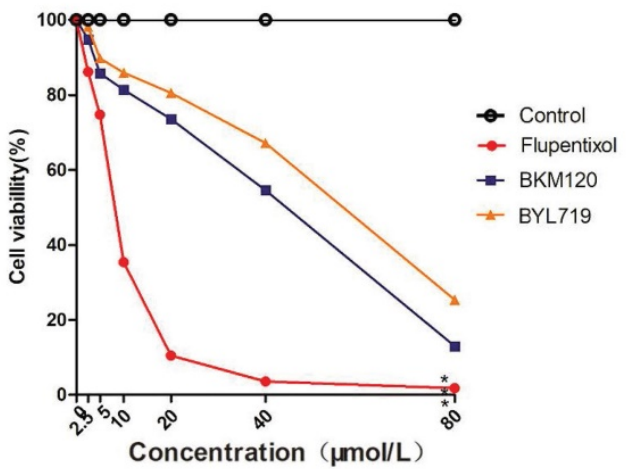

H661

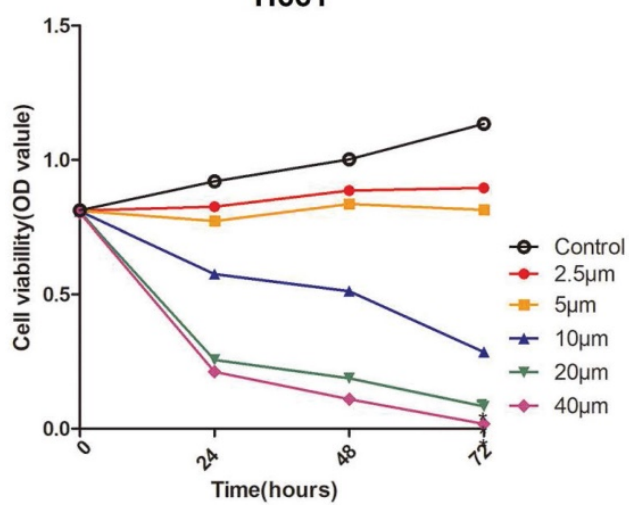

H661

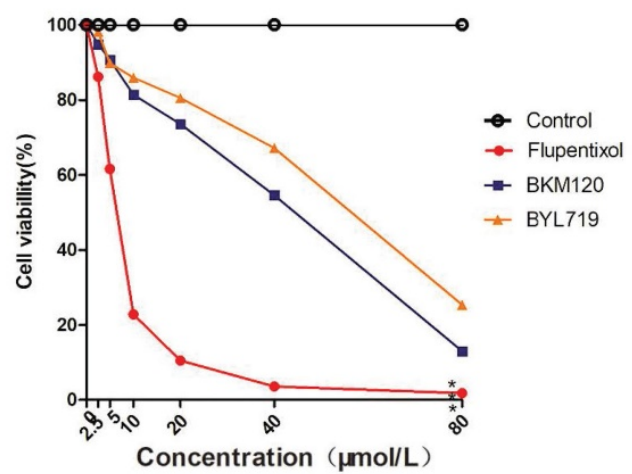

Figure 2. Flupentixol decreases lung cancer cell viability. (A) Flupentixol (2.5, 5, 10, 20, or $40 \mu \mathrm{M})$ inhibited the viability of four NSCLC cell lines (H661, A549, H520 and SK-SEM-1) in a dose-dependent manner but had little effect on BEAS-2B cells. The cells were treated with flupentixol for 24 hours, and MTT assays were performed to measure cell viability. (B) H661 and A549 cells were treated with increasing concentrations of flupentixol $(2.5,5,10,20$, or $40 \mu \mathrm{M})$ for 24,48 , and 72 hours. The inhibitory effect of flupentixol in lung cancer cells was in a dose- and time-dependent manner. (C) The 24-hour cytotoxicity of flupentixol was stronger than those of two known PI3K inhibitors (BYL719 and BKM120) in A549 and $\mathrm{H} 661$ cells. 
(A)

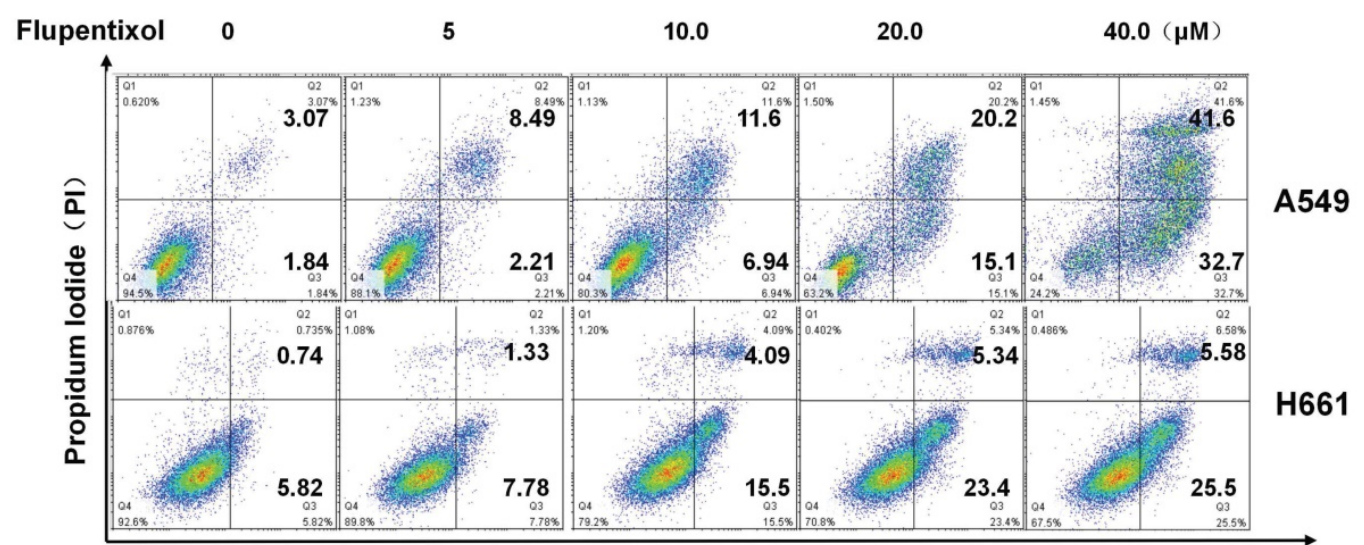

Annexin V-FITC

(B)

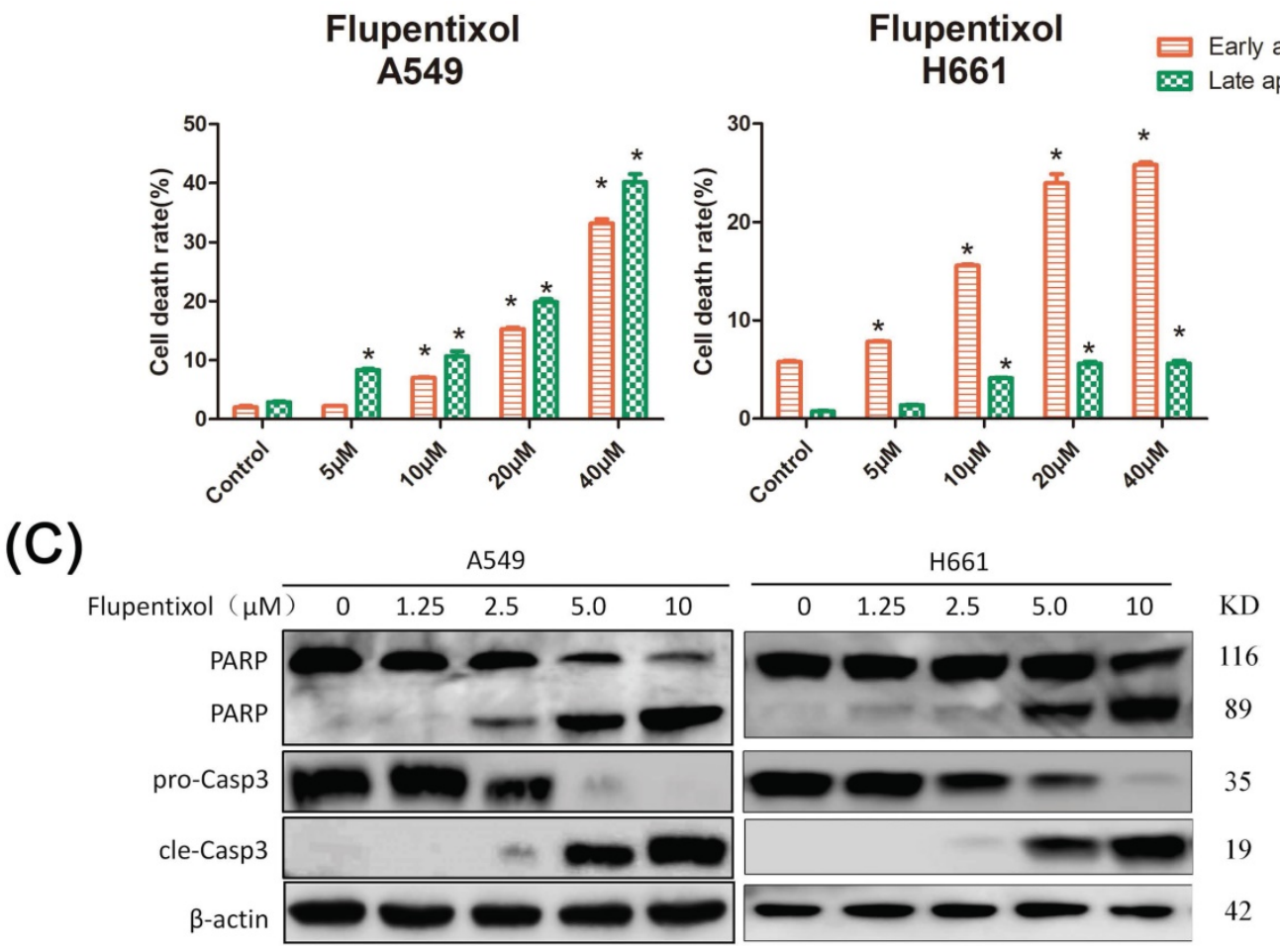

Figure 3. Flupentixol induces apoptosis in lung cancer cells. (A) Flupentixol induced apoptosis in A549 and H661 lung cancer cells. A549 and H661 cells were treated with flupentixol at 5, 10,20 or $40 \mu \mathrm{M}$ for 24 hours. Apoptosis was measured by Annexin V/PI staining and flow cytometry. (B) The quantitative results of panel A. (C) Flupentixol induced the cleavage of caspase- 3 and PARP in lung cancer cells, as measured by Western blotting. A549 and $\mathrm{H} 661$ cells were treated with flupentixol ( $1.25,2.5,5$, or $10 \mu \mathrm{M})$ for 24 hours.

\section{Discussion}

Nonsmall-cell lung cancer (NSCLC) is the most common pathological type of lung cancer. The treatment of NSCLC has changed the distribution of the histological subtypes to defined molecular targets. EGFR gene mutations, MET amplification, EML4-ALK rearrangements are widely recognized alterations involved in the pathobiology with clinical impact for the NSCLC treatment. Recently, new oncogenic gene alterations have been identified in
NSCLC, including HER2 mutations, BRAF mutations, and alterations of the PIK3/AKT/mTOR pathway [16]. The PI3K/AKT pathway is activated in many cancers and plays a pivotal role in both initiation and progression of NSCLC [17]. Mutations in the PIK3CA gene encoding the class I PI3K p110a, are identified in $3.7 \%$ of the total cohort of 1,144 NSCLC patients [18]. PIK3CA was mutated in $15 \%$ of 132 advanced NSCLC patients with EGFR mutation treated with gefitinib [8]. PIK3CA amplification was frequently found in the Chinese NSCLC populations [19]. 
(A)
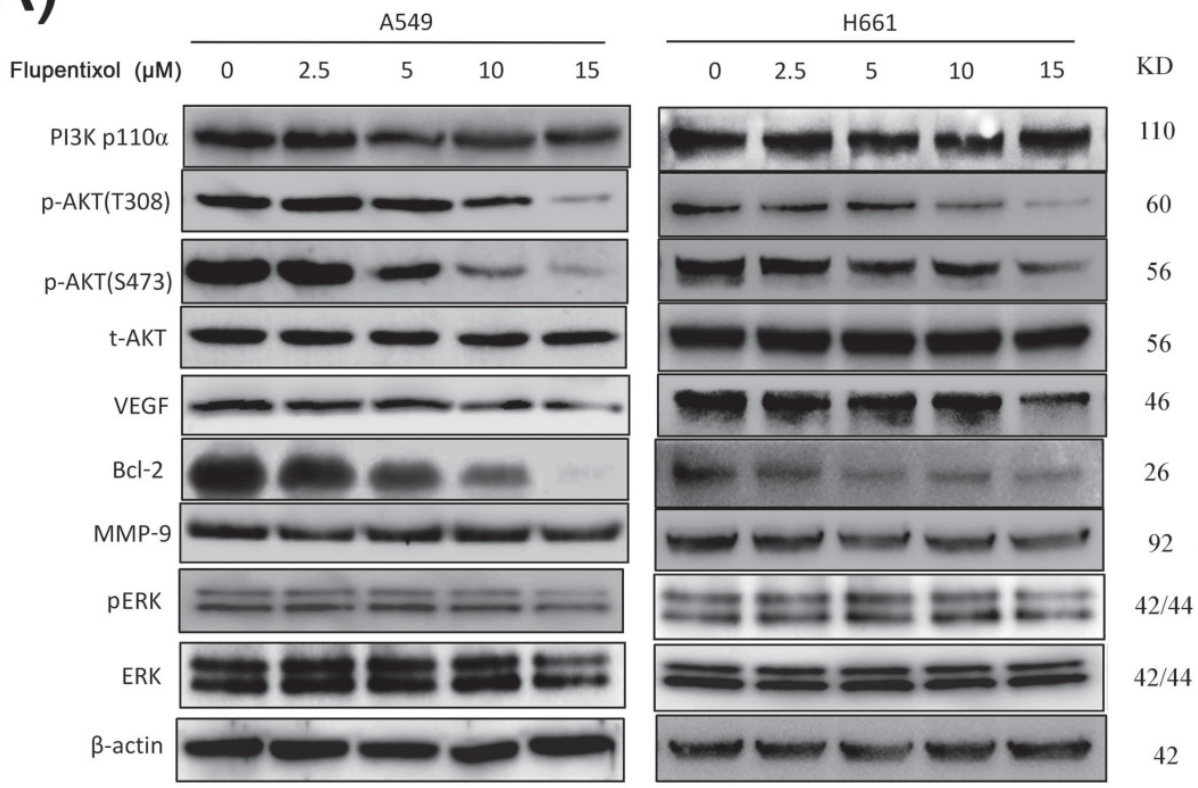

(B)
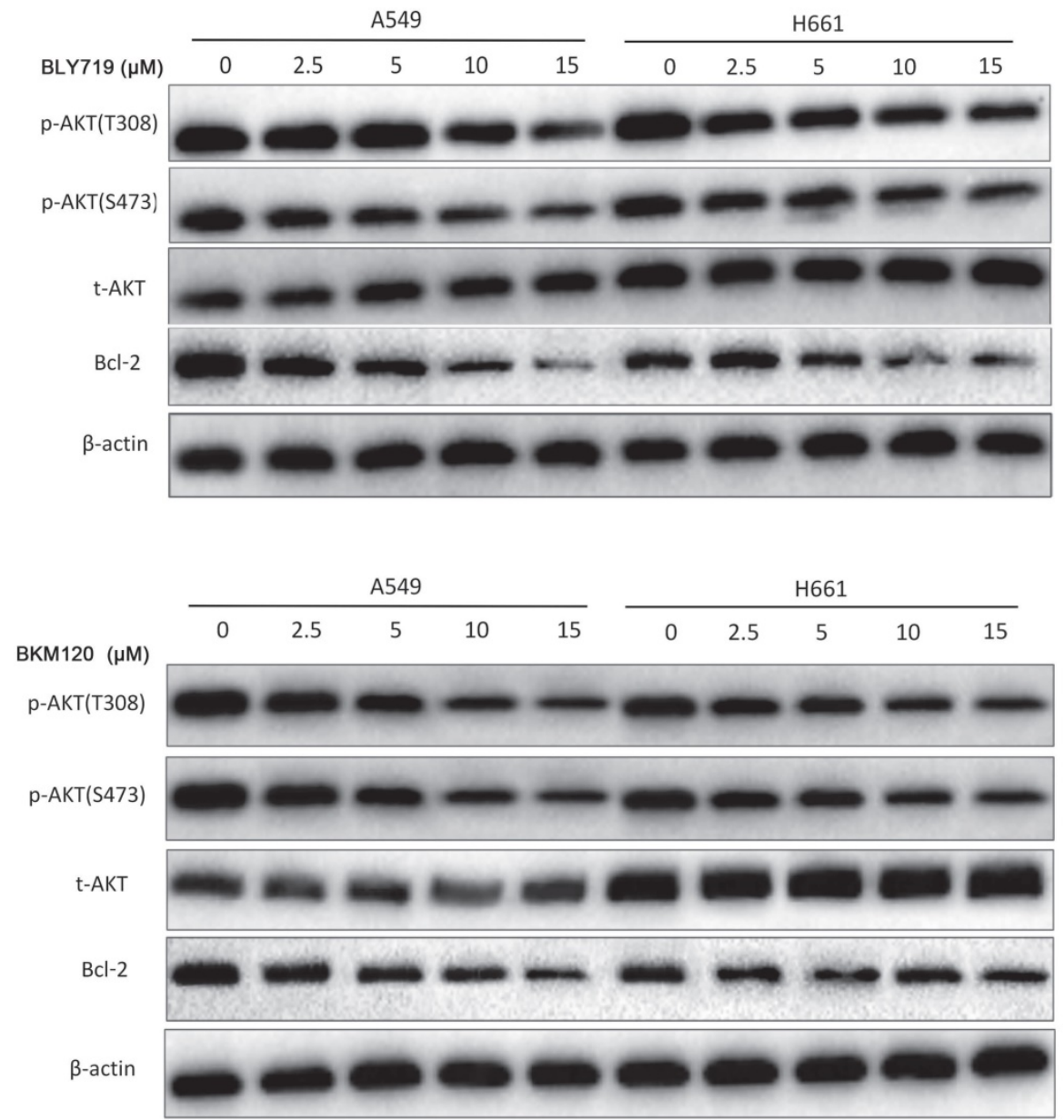

Figure 4. Flupentixol specifically inhibits the PI3K/AKT pathway in lung cancer cells. (A) The expression levels of $\mathrm{p}-\mathrm{AKT}$ (S473 and T308) and Bcl-2 were decreased by flupentixol in a dose-dependent manner. A549 and $\mathrm{H} 661$ cells were treated with increasing concentrations of flupentixol $(2.5,5,10$ or $15 \mu \mathrm{M})$ for 24 hours. Flupentixol had no effect on PI3K p110a, t-AKT, p-ERK, t-ERK, VEGF, or MMP-9. (B) The inhibitory effects of two known PI3K inhibitors (BYL719 and BKMI20) on the expression of p-AKT, t-AKT, and $\mathrm{Bcl}-2$ in $\mathrm{A} 549$ and $\mathrm{H} 661$ cells. 
(A)

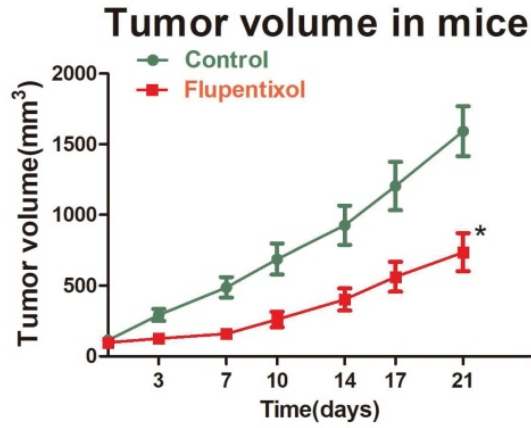

(C)

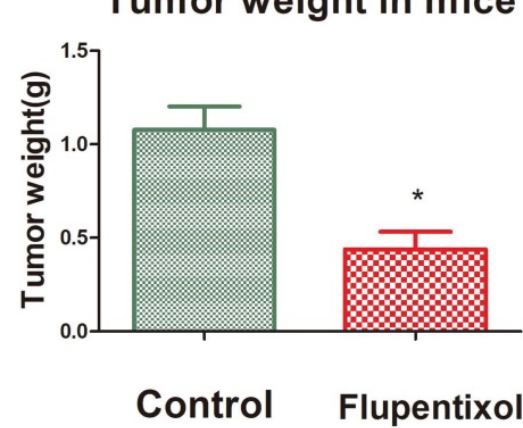

(B)

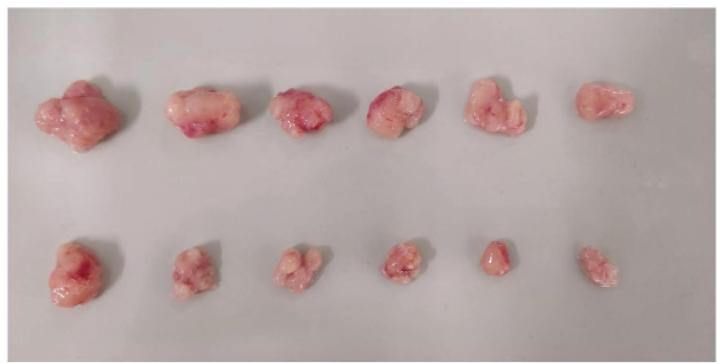

(D)

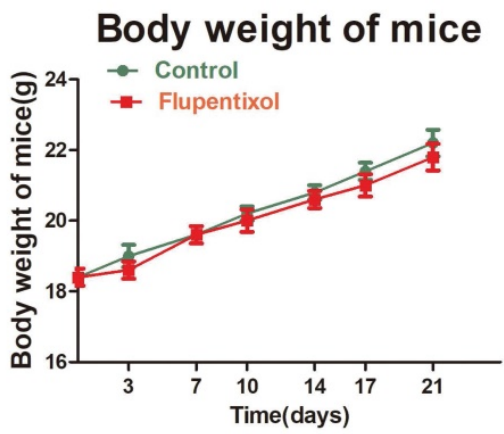

Figure 5. Flupentixol suppresses A549 tumor growth in nude mice. (A) Flupentixol significantly inhibited A549 tumor growth in nude mice. A549 cells were subcutaneously inoculated in BALB/C nude mice to allow growth to approximately $70-80 \mathrm{~mm}^{3}$. Flupentixol (40 mg/kg daily) was administrated (i.g.) daily for 21 days. (B) The tumor mass harvest at the end of the experiment. (C) The average tumor weights from the flupentixol treatment group and the negative control group. (D) Flupentixol had no significant effect on the mouse body weights.

The phosphatidylinositol 3-kinases (PI3Ks) are a family of lipid kinases. The class I PI3Ks among three subclasses are heterodimeric protein complexes composed of a p110a catalytic subunit and a p85 regulatory subunit, primarily involved in the pathogenesis of human cancers [17]. Furthermore, the tumorigenic potential of p110a mutations was well validated [20]. In contrast, no oncogenic mutations were identified in other catalytic subunits, p110 $\beta$, p110y, or p1108. The activation of the PI3K/AKT signaling pathway leads to increases in cell proliferation and survival. AKT promotes cell growth and survival by phosphorylating many cellular targets, which trigger distinct downstream signaling events [21]. Moreover, the aberrant activation of the PI3K/AKT pathway plays a role in the development of resistance to chemotherapy and acquired resistance to EGFR inhibitors [5]. Thus, PI3K is a promising therapeutic target for lung cancer.

Several PI3K inhibitors are currently undergoing evaluation in preclinical and clinical studies [22]. These PI3K inhibitors include pan and selective PI3K inhibitors and dual PI3K and mTOR inhibitors. Idelalisib, also known as CAL-101, was approved in the United States and European Union for the treatment of three indolent B-cell neoplasms in 2014 [23]. Buparlisib, an oral pan-PI3K inhibitor, has shown preclinical anti-tumor activity. The phase I study of buparlisib in adult Chinese patients with advanced solid tumors established a recommended dose of 100 $\mathrm{mg}$ /day and showed an acceptable safety profile and evidence of efficacy [24]. The phase I study of buparlisib combined with carboplatin and paclitaxel for advanced solid tumor patients (NCT01297452) showed the notable preliminary activity in PTEN-deficient tumors [25]. Buparlisib reverted the aggressive phenotype by reducing metalloproteinase production in the xenograft model of squamous cell carcinoma of the lung (SQCLC) [26]. A phase II cluster study of BYL719, a specific PI3Ka inhibitor, in patients with PIK3CA gene mutation/amplification in Chinese patients with advanced nonsmall cell lung cancer is ongoing [27]. To date, PI3K inhibitors have not been approved for lung cancer yet.

Flupenthixol is an antipsychotic medication used to treat schizophrenia and/or depression in a dose range of $0.5-3 \mathrm{mg} /$ tablet for decades [28]. As a strong and selective dopamine receptor antagonist, flupenthixol inhibited drug transport and reverse drug resistance mediated by the human multidrug transporter P-glycoprotein (P-gp) [29]. Recent studies showed that flupentixol may improve chemotherapy sensitivity and overcome drug resistance mediated by downregulation of the mutant MDR1 [29]. Flupentixol 
reversed multidrug resistance and improved chemosensitivity in murine fibrosarcoma cells [13].

Here, we demonstrated that flupentixol possesses cytotoxicity in all pathological types of NSCLC cell lines, including H661 (large cell lung cancer), A549 (adenocarcinoma), NCI-H520 and SK-SEM-1 (squamous cell carcinoma). Flupentixol showed the strongest inhibitory effect on A549 and H661, while it had little toxicity in normal lung bronchial epithelial cells. Compared to two known PI3K inhibitors, BYL719 and BKM120, flupentixol showed more potent cytotoxicity in A549 and H661 cells. The pan-PI3K inhibitor BKM120 showed a significant cytotoxic activity on various tumor cell lines, including lung cancer, and a strong synergistic antiproliferative effect combined with chemotherapy [30]. BYL719, a specific PI3Ka inhibitor, combined with MEK1/2 inhibitor showed a synergistic effect on lung cancer A549 cell line in vivo and in vitro [31]. We previously reported that flupenthixol $(20 \mu \mathrm{M})$ exhibited anticancer activity in both HCC827 and H1975 lung cancer cells harboring the EGFR T790M mutation [12]. Flupentixol dihydrochloride is a thiophene drug with a piperazine side chain. The pharmacological structure of thiophene was assessed for anti-inflammatory and anti-cancer activities [32]. We speculate that the anticancer effect of flupentixol dihydrochloride may be related the structure of thiophene. More structure and function analyses will be required to develop better compounds derived from flupenthixol.

By phosphorylating a large number of downstream targets, AKT promotes several important processes, including cell proliferation, survival, angiogenesis, invasion, and metastasis [33]. Flupentixol significantly inhibited the phosphorylation of AKT at both T308 and S473 sites in a concentration-dependent manner but had no effect on the total expression of AKT ( $\mathrm{t}-\mathrm{AKT})$. Moreover, it had no inhibitory effect on ERK, suggesting that flupentixol may be a specific inhibitor of the PI3K/AKT pathway. Flupentixol decreased the Bcl-2 protein expression levels in both $\mathrm{H} 661$ and A549 cell lines. Bcl-2 is a key pro-survival protein whose expression is upregulated by AKT [34]. We confirmed that flupentixol induces the cleavage of caspase 3 and PARP in both H661 and A549 lung cancer cell lines. The inhibitory effect of flupentixol on p-AKT/Bcl-2 was stronger than those of the known inhibitors (BYL719 and BLM120).

Flupentixol showed significant antitumor effects on lung cancer A549 xenograft in nude mice by daily oral administration. The pharmacokinetics analysis on flupentixol showed that absorption rate could be obtained from the oral film formulation and the relative bioavailability in humans was approximately $55 \%$ [35]. The time to reach peak concentrations for tablet formulations ranged from 3 to 8 hours and the elimination half-life $\left(\mathrm{t}^{1 / 2} \beta\right)$ was approximately 35 hours [36]. This finding suggested that flupentixol had a reliable oral activity. Moreover, it should be noted that flupentixol is slowly absorbed with a relatively long half-life, suggesting that oral administration of flupentixol is safe and tolerable. The reported side-effects include renal insufficiency, hypertension and, QT prolongation [37]. It is necessary to conduct further experimental research on the side effects of flupentixol.

Inhibition of the PI3K/AKT pathway may overcome chemosensitivity in NSCLC. Recent preclinical and clinical data on PI3K pathway inhibitors in NSCLC, either as monotherapy approach or in combination with chemotherapy or with drugs, was effective [38]. The combination of buparlisib and carboplatin or paclitaxel was well tolerated and exhibited notable effects against tumors [39]. Therefore, the combinations of flupentixol with other chemotherapies may exhibit synergistic efficacy.

In summary, we found that flupentixol could be docked to the PI3K $\alpha$ protein. Flupentixol can specifically inhibit the PI3K/AKT pathway and survival of lung cancer cells in vitro and in vivo. As an old drug, flupentixol may become a targeted drug for lung cancer with overactivation of the PI3K/AKT pathway.

\section{Acknowledgments}

This study was supported in part by grants from the National Nature Science Foundation of China (81830087, U1602221 and 31771516 to Chen, C.), the medical reserve personnel training program from the Health and Family Planning Commission of Yunnan province (H-201609 to Dong, C), Health Science and Technology project of Yunnan province (2018NS0052 to Dong, C), and talent innovation project in Xishan District, Kunming.

\section{Author Contributions}

C.S.C., and M.C.L. designed the study. C.D, Y.C, and K.K performed the experiments, and collected and interpreted the data. C.D., Y.C, C.S.C., H.J.L., and M.C.L. wrote and revised the manuscript. All authors discussed and commented on the manuscript. G.M.L. and X.M.W. provided material support and supervised the study.

\section{Competing Interests}

The authors have declared that no competing interest exists. 


\section{References}

1. Torre LA, Bray F, Siegel RL et al. Global cancer statistics, 2012. Ca A Cancer Journal for Clinicians. 2015; 65: 87-108.

2. Mayekar MK, Bivona TG. Current landscape of targeted therapy in lung cancer. Clinical Pharmacology \& Therapeutics. 2017; 102.

3. Tan CS, Gilligan D, Pacey S. Treatment approaches for EGFR-inhibitor-resistant patients with non-small-cell lung cancer. Lancet Oncology. 2015; 16: e447-e59.

4. Azzato EM, Deshpande C, Aikawa V et al. Rare Complex Mutational Profile in an ALK Inhibitor-resistant Non-small Cell Lung Cancer. Anticancer Research. 2015; 35: 3007

5. Fumarola C, Bonelli MA, Petronini PG, Alfieri RR. Targeting $\mathrm{PI} 3 \mathrm{~K} / \mathrm{AKT} / \mathrm{mTOR}$ pathway in non small cell lung cancer. Biochemical Pharmacology. 2014; 90: 197-207.

6. Rd BH. Overcoming acquired resistance to anticancer therapy: focus on the PI3K/AKT/mTOR pathway. Cancer Chemotherapy \& Pharmacology. 2013; 71: $829-42$.

7. Gadgeel SM, Wozniak A. Preclinical rationale for PI3K/Akt/mTOR pathway inhibitors as therapy for epidermal growth factor receptor inhibitor-resistant non-small-cell lung cancer. Clinical Lung Cancer. 2013; 14: 322-32.

8. Lim SM, Kim HR, Cho EK et al. Targeted sequencing identifies genetic alterations that confer primary resistance to EGFR tyrosine kinase inhibitor (Korean Lung Cancer Consortium). Oncotarget. 2016; 7: 36311-20.

9. C P, M C, MÁ M et al. PTEN and PI3K/AKT in non-small-cell lung cancer. Pharmacogenomics. 2015; 16: 1843-62.

10. Massacesi C, Tomaso ED, Urban P et al. PI3K inhibitors as new cancer therapeutics: implications for clinical trial design. Oncotargets \& Therapy. 2016; 9: 203-10.

11. ChB ML-WM. Treatment of depression with flupenthixol in terminally ill patients. European Journal of Cancer Care. 2010; 3: 133-4.

12. $\mathrm{Li} \mathrm{H}$, Tong CW, Leung $\mathrm{Y}$ et al. Identification of Clinically Approved Drugs Indacaterol and Canagliflozin for Repurposing to Treat Epidermal Growth Factor Tyrosine Kinase Inhibitor-Resistant Lung Cancer. Frontiers in Oncology. 2017; 7: 288.

13. Fan D, Poste G, Seid C et al. Reversal of multidrug resistance in murine fibrosarcoma cells by thioxanthene flupentixol. Investigational New Drugs. 1994; 12: 185.

14. Li H, Leung KS, Ballester PJ, Wong MH. istar: a web platform for large-scale protein-ligand docking. Plos One. 2014; 9: e85678.

15. Li H, Leung KS, Wong MH. idock: A multithreaded virtual screening tool for flexible ligand docking. IEEE Symposium on Computational Intelligence in Bioinformatics \& Computational Biology. 2012; 2012. p. 77-84.

16. Thomas A, Liu SV, Subramaniam DS, Giaccone G. Refining the treatment of NSCLC according to histological and molecular subtypes. Nature Reviews Clinical Oncology. 2015; 12: 511.

17. Thorpe LM, Yuzugullu H, Zhao JJ. PI3K in cancer: divergent roles of isoforms, modes of activation and therapeutic targeting. Nature Reviews Cancer. 2015; 15: 7-24.

18. Scheffler M, Bos M, Gardizi M et al. PIK3CA mutations in non-small cell lung cancer (NSCLC): Genetic heterogeneity, prognostic impact and incidence of prior malignancies. Oncotarget. 2015; 6: 1315-26.

19. Ji M, Guan $\mathrm{H}$, Gao $\mathrm{C}$ et al. Highly frequent promoter methylation andPIK3CAamplification in non-small cell lung cancer (NSCLC). BMC Cancer. 2011; 11: 1-11.

20. Samuels Y, Waldman T. Oncogenic Mutations of PIK3CA in Human Cancers. Cell Cycle. 2004; 3: 1221-4.

21. Balzano D, Fawal MA, Velázquez JV et al. Alternative Activation Mechanisms of Protein Kinase B Trigger Distinct Downstream Signaling Responses. Journal of Biological Chemistry. 2015; 290: 24975-85.

22. Wang X, Ding J, Meng LH. PI3K isoform-selective inhibitors: next-generation targeted cancer therapies. 中国药理学报. 2015; 36: 1170-6.

23. Yang $Q$, Modi $P$, Newcomb $T$ et al. Idelalisib: First-in-Class PI3K Delta Inhibitor for the Treatment of Chronic Lymphocytic Leukemia, Small Lymphocytic Leukemia, and Follicular Lymphoma. Clinical Cancer Research. 2015; 21: 1537-42.

24. Wu YL, Zhang LI, Trandafir L et al. Phase I Study of the Pan-PI3K Inhibitor Buparlisib in Adult Chinese Patients with Advanced Solid Tumors. Anticancer Research. 2016; 36: 6185.

25. Hyman DM, Snyder AE, Carvajal RD et al. Parallel phase Ib studies of two schedules of buparlisib (BKM120) plus carboplatin and paclitaxel (q21 days or q28 days) for patients with advanced solid tumors. Cancer Chemotherapy \& Pharmacology. 2015; 75: 1-9.

26. Bonelli MA, Cavazzoni A, Saccani F et al. Inhibition of PI3K pathway reduces invasiveness and epithelial-to-mesenchymal transition in squamous lung cancer cell lines harboring PIK3CA gene alterations. Mol Cancer Ther. 2015; 14: 1916-27.

27. Zhou Q, Zhang XC, Peng B et al. A phase II cluster study of single agent AUY922, BYL719, INC280, LDK378, and MEK162 in Chinese patients with advanced non-small cell lung cancer (NSCLC). J Clin Oncol. 2014; 32: 15 Suppl.

28. Abdelbary A, Bendas ER, Ramadan AA, Mostafa DA. Pharmaceutical and Pharmacokinetic Evaluation of a Novel Fast Dissolving Film Formulation of Flupentixol Dihydrochloride. Aaps Pharmscitech. 2014; 15: 1603-10.

29. Dey S, Hafkemeyer P, Pastan I, Gottesman MM. A Single Amino Acid Residue Contributes to Distinct Mechanisms of Inhibition of the Human Multidrug
Transporter by Stereoisomers of the Dopamine Receptor Antagonist Flupentixol. Biochemistry. 1999; 38: 6630-9.

30. Maira SM, Pecchi S, Huang A et al. Identification and characterization of NVP-BKM120, an orally available pan-class I PI3-kinase inhibitor. Molecular Cancer Therapeutics. 2012; 11: 317.

31. Bo MK, Jho EH, Bae YH et al. BYL719, a selective inhibitor of phosphoinositide 3-Kinase $a$, enhances the effect of selumetinib (AZD6244, ARRY-142886) in KRAS-mutant non-small cell lung cancer. Investigational New Drugs. 2015; 33: $12-21$

32. Rakesh KS, Jagadish S, Swaroop TR et al. Anti-Cancer Activity of 2,4-Disubstituted Thiophene Derivatives: Dual Inhibitors of Lipoxygenase and Cyclooxygenase. Medicinal Chemistry. 2015; 11: -.

33. Martini M, De Santis MC, Braccini L et al. PI3K/AKT signaling pathway and cancer: an updated review. Annals of Medicine. 2014; 46: 372-83.

34. Kim SY, Yoo SJ, Ronnett GV et al. Odorant Stimulation Promotes Survival of Rodent Olfactory Receptor Neurons via PI3K/Akt Activation and Bcl-2 Expression. Molecules \& Cells. 2015; 38: 535.

35. Jørgensen A. Pharmacokinetic studies in volunteers of intravenous and oral cis (Z)-flupentixol and intramuscular cis (Z)-flupentixol decanoate in Viscoleo. European Journal of Clinical Pharmacology. 1980; 18: 355-60.

36. Walter S, Bauer S, Roots I, Brockmöller J. Quantification of the antipsychotics flupentixol and haloperidol in human serum by high-performance liquid chromatography with ultraviolet detection. Journal of Chromatography B Biomedical Sciences \& Applications. 1998; 720: 231-7.

37. Marcussen M, Polcwiartek C, Anna Mills EH et al. Abnormal serum potassium levels and 6-month all-cause mortality in patients co-treated with antipsychotic and diuretic drugs - A Danish register-based cohort study. Eur Neuropsychopharmacol. 2018: S0924977X18301688-

38. Heavey S, O'Byrne KJ, Gately K. Strategies for co-targeting the PI3K/AKT/mTOR pathway in NSCLC. Cancer Treatment Reviews. 2014; 40: 445 .

39. Hyman DM, Snyder AE, Carvajal RD et al. Parallel phase Ib studies of two schedules of buparlisib (BKM120) plus carboplatin and paclitaxel (q21 days or q28 days) for patients with advanced solid tumors. Cancer Chemotherapy \& Pharmacology. 2015; 75: 747-55. 